had a highly variable cough frequency during the first 8 weeks of treatment. There was no evidence for an effect of isoniazid resistance, cavitary disease, smear status or smoking on early rates of cough resolution, although there was a trend towards relatively higher cough frequencies in smokers than non-smokers at the end of treatment $(p=0.100)$.

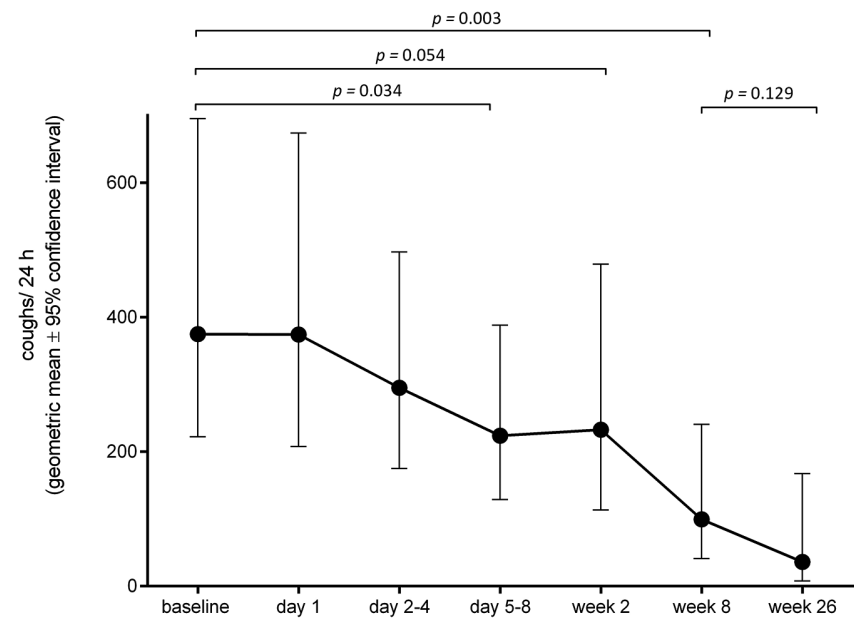

Abstract S37 Figure 1 Objectively-measured cough frequency during treatment of pulmonary tuberculosis

Conclusions Objective cough frequency measurement is feasible in tuberculosis and could provide a novel biomarker of treatment response.

\section{S38 PREDICTIVE ACCURACY AND CLINICAL IMPACT OF XPERT MTB/RIF FOR THE DIAGNOSIS OF SPUTUM SMEAR-NEGATIVE PULMONARY TUBERCULOSIS USING BRONCHOALVEOLAR LAVAGE FLUID}

${ }^{1} W$ Ho, ${ }^{2} \mathrm{DW}$ Connell, ${ }^{2} \mathrm{~A}$ Singanayagam, ${ }^{2} \mathrm{~A}$ Singanayagam, ${ }^{2} \mathrm{H}$ Donaldson, ${ }^{2} \mathrm{OM}$ Kon. ${ }^{1}$ Imperial College School of Medicine, Imperial College London, London, UK; ${ }^{2} S t$. Mary's Hospital, Imperial College Healthcare NHS Trust, London, UK

\subsection{6/thoraxjnl-2015-207770.44}

Introduction Sputum smear-negative pulmonary tuberculosis (TB) is increasingly prevalent with bronchoalveolar lavage (BAL) frequently used for diagnostic sampling. Direct molecular testing has reported higher sensitivities compared to smear microscopy. This study aims to assess the predictive accuracy and clinical impact of Xpert MTB/RIF; a PCR-based cartridge assay used to identify M.tb in BAL fluid samples.

Methods A retrospective evaluation of adult patients $(n=293)$ with suspected pulmonary TB who underwent BAL in a tertiary centre in London between January 2011 and December 2014 were collected. MTB/RIF, smear microscopy, and liquid culture were performed on all sets of BAL fluid. The impact of MTB/ RIF on time to TB diagnosis and anti-TB treatment initiation were recorded as markers of clinical impact.

Results 57/293 (19.5\%) patients had BAL culture-positive TB for which a significantly higher proportion had positive MTB/RIF results compared to smear microscopy $(77.2 \%$, 95\% CI 63.8\%$86.8 \%$ vs. $38.6 \%$, $95 \%$ CI $26.3 \%-52.4 \%$; p < 0.001$)$. The specificity of MTB/RIF was $95.7 \%(92.1 \%-97.8 \%)$ with a negative predictive value (NPV) of $94.6 \% \quad(90.7 \%-97.0 \%)$. 22/57 (38.6\%) culture-positive patients had negative smear microscopy results but positive MTB/RIF results.
90/293 (30.7\%) patients were clinically-diagnosed and treated for pulmonary TB. In this subgroup, MTB/RIF again outperformed smear microscopy in terms of sensitivity $(54.2 \%$; $95 \%$ CI $43.7 \%-64.3 \%$ vs. $27.1 \%$, 95\% CI $18.8 \%-37.3 \%$; p < $0.001)$. The specificity of MTB/RIF was $81.6 \%$ (80.0\%-86.2\%).

The use of MTB/RIF provided a significant advantage in time to $\mathrm{TB}$ diagnosis in culture-positive patients as compared to smear microscopy (1 days; IQR 0-2 days versus 10 days; IQR 0-15 days; $p<0.05)$. In a specific cohort of smear-negative cultureconfirmed TB patients, MTB/RIF reduced the time to TB diagnosis from an average of 14 days (95\% CI 12-16 days) to 1 day (95\% CI 0-2 days; $\mathrm{p}<0.05)$.

There was no statistical difference in time to anti-TB treatment initiation between those who were either smear microscopy positive and/or MTB/RIF positive in culture-positive patients ( 1 day; IQR 0-3 days versus 1 day; IQR $0-2$ days; $p=$ $0.164)$.

Conclusion MTB/RIF used in BAL samples had a higher and more rapid diagnostic accuracy compared to smear microscopy and could replace routine smear microscopy in pulmonary $\mathrm{TB}$ diagnosis.

\section{S39 PRELIMINARY RESULTS OF A LATENT TUBERCULOSIS SCREENING AND TREATMENT PROJECT AND THE ROLE OF TB SERVICES IN SECONDARY CARE}

MGK Burman, G Ahmed, JL Potter, VLC White, N Jayasekera, H Kunst. Barts Health NHS Trust, London, UK

\subsection{6/thoraxjnl-2015-207770.45}

Introduction Since July 2014, the London Borough of Newham has offered latent tuberculosis (TB) screening to all recent migrants (residing in the UK less than 5 years), aged 16-50 years, from countries with a TB incidence of greater than 150/ 100000 cases/year. All migrants are offered an interferon gamma-release assay (IGRA) when registering with a general practitioner. Active TB is excluded by the GP using chest radiography, blood tests and clinical examination. All IGRA positive patients are tested for HIV, Hepatitis B and C. All patients without underlying liver disease, Hepatitis B, C or HIV infection and those who are not immunosuppressed are offered treatment for LTBI with Rifampicin and Isoniazid for 3 months in primary care. Patients with positive results not meeting the above exclusion criteria are referred to the local secondary care service using a standardised referral protocol.

We conducted a retrospective study reviewing records of all patients referred to secondary care from the LTBI screening programme.

Results From July 2014 to March 2015, a total of 5683 patients were offered screening. 3272 proceeded to IGRA testing of which 866 were positive. Of these patients, 138 were referred to the TB clinic. The most common reasons for referral were symptoms suggestive of active TB (26\%), abnormal liver function tests (19\%) before and after initiation of treatment, an abnormal chest radiograph (CXR) (10\%), Pregnancy or breastfeeding (9\%), Hepatitis B or C infection (7\%) or previously treated latent or active TB (7\%). Of those referred, 11 patients were found to have active disease. 6 patients had mediastinal lymphnode TB, 4 pulmonary and one patient had TB of the knee.

Conclusion Screening for latent tuberculosis in primary care has identified a significant of number of cases of active Tuberculosis, particularly mediastinal TB. 